\title{
An Adaptive Phase Alignment Algorithm for Cartesian Feedback Loops
}

A system with a feedback loop typically experiences a delay between its forward and feedback paths. This phenomenon, commonly observed in power amplifiers, can be addressed with a Cartesian feedback linearization loop, which seeks to synchronize the forward and feedback paths for optimal performance. This article presents an adaptive phase alignment algorithm based on the vectorial product of forward and feedback Cartesian inphase/quadrature (I/Q) signals. Phase misalignments between forward and feedback paths smaller than $\pm \pi / 2$ can be corrected yielding phase errors smaller than $0.035 \mathrm{rad}$. The algorithm has been successfully implemented and tested on fixed point digital signal processing (DSP) hardware to linearize envelope elimination and restoration (EER) power amplifiers.

\section{PRACTICAL PROBLEMS OF DIGITAL CARTESIAN FEEDBACK LOOPS}

Linearization of a power amplifier is commonly achieved with a Cartesian feedback loop. Basically, the linearization process compares the baseband Cartesian inputs with baseband Cartesian signals downconverted from the amplifier's output. The resulting error is filtered, integrated, and fed to the radio frequency (RF) power amplifier. In effect, the Cartesian feedback loop predistorts the source signal to compensate for the amplifier's distortion. Moreover, it easily compensates aging and temperature drifts in the amplifier's gain and phase characteristics [1]. Figure 1 shows a Cartesian feedback loop topology, which is applied to a high efficiency power amplifier (HEPA).
In theory, Cartesian loops for amplifier linearization operate on ideal, phase aligned, forward and feedback signals that are completely synchronized. In practice, however, the systems or devices that process these signals add some delay. The phase misalignment problem arises from the feedback and forward signals' lack of synchronization. As a result, signals that are subtracted using digital or analog techniques are phase delayed. Phase misalignments in Cartesian feedback loops can lead to error vector magnitude (EVM) degradation and loop instability [2]. In addition, in real-life hardware, these phase misalignments change with variables such as temperature and frequency.

Analog feedback loops do not usually add a meaningful delay to the processed signals, since most of the delay results from the propagation time in the system components and is small when compared to the period of the fundamental frequency (when the system works below microwave frequencies). However, when the feedback loop is partly analog and partly digital, the sampling period becomes a problem. Since the forward and feedback signal goes from the digital to the analog domain and vice versa, it is necessary to use both digital to analog converters (DACs) and analog to digital converters (ADCs). They add a delay that is equivalent to an integer number of samples, since they usually buffer the input signal. Even though the sampling frequency may be very high, the delay that is added by the converters can make the loop unstable.

However, feedback digital systems provide important benefits. For instance, the comparator used in analog Cartesian loops is replaced by a simple digital subtract, so the comparator does not add distortion to the system. The gain and bandwidth of the loop could be adaptively updated until their optimal values were determined. Moreover, these values would have a high accuracy, since they are digital, so the performance of the linearization process would improve. This accuracy would be difficult to achieve with analog elements. Furthermore, there are many other elements that can be digitally implemented, avoiding the distortion generated by their analog counterparts. Since the loop is digital, it would be also possible to design and easily implement, in a digital architecture, any processing block that

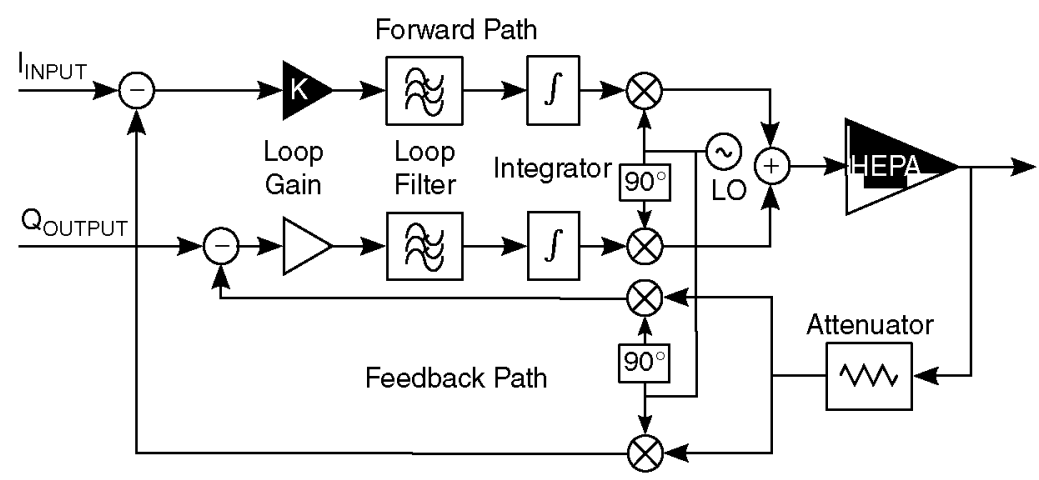

[FIG1] Linearization by Cartesian feedback loop technique. 
improves the linearization process. For instance, an image band rejection algorithm could be added to the initial design. Then, the image band that would appear at the $\mathrm{ADC}$ inputs could be rejected. Only the desired signal would affect the loop.

Digital feedback loops provide the designer much flexibility during design and implementation stages. Many analog elements can be replaced by digital ones, removing nonlinearities and making the linearization process more efficient. Therefore, it is desirable to keep the benefits of digital feedback systems and to determine a solution to the phase misalignment issue as proposed in this article.

\section{PHASE ALIGNMENT ADAPTIVE ALGORITHM PRINCIPLES}

Forward and feedback signals of Cartesian loops can each be modeled by a vector. The phase angle between these two vectors determines the phase difference between both forward and feedback signals [3]. If these forward and feedback vectors are misaligned, some adjustment of their phase angle can be implemented at baseband by feeding a fraction of the I-channel signal into the Q-channel and vice versa [4]. However, such an alignment method is not adaptive and unsuitable for correcting phase misalignment resulting from aging, temperature, and other factors. In the next few sections, a novel adaptive phase alignment algorithm based on the vectorial product of Cartesian loop forward and feedback signals is proposed. It does not exhibit the previously related problems. The vectorial product of Cartesian loop forward and feedback signals can be obtained with

$$
V P=I \cdot Q^{\prime}-Q \cdot I^{\prime}=r \cdot r^{\prime} \sin (\theta),
$$

where $I$ and $Q$ are the forward signals of the Cartesian loop, $I^{\prime}$ and $Q^{\prime}$ are the feedback signals of the Cartesian loop, $r$ is the vector modulus of the forward signal $(|I+j Q|), r^{\prime}$ is the vector modulus of the feedback signal $\left(\left|I^{\prime}+j Q^{\prime}\right|\right)$, and $\theta$ is the phase difference between the two vectors.

The proposed algorithm determines of the phase difference between both signals when it is within the range $-\pi / 2<\theta<\pi / 2$ [5]. The algorithm is based on reducing the vectorial product of the forward and the feedback vector by means of a least mean squares (LMS) algorithm. An appropriate forward phase shift, obtained by the LMS algorithm, is applied to the forward path; this action compensates the phase difference between forward and feedback signals introduced by the Cartesian loop hardware.

The step-size value of the LMS algorithm is also adaptive in this system. At the beginning of the process the stepsize of the LMS algorithm is set high, allowing a fast convergence around the final optimum value. The step-size is reduced as the algorithm converges: the smaller the vectorial product is, the smaller the step-size value is set to achieve a better resolution.

\section{PHASE ALIGNMENT ADAPTIVE ALGORITHM IMPLEMENTATION}

The proposed phase alignment algorithm is illustrated in Figure 2. The $I / Q$ signals are generated by the "signal generator" block. Block I is a phase shifter that shifts forward $\theta$ rad the forward signal [6]. The phase shifter can be accurately implemented either in the feedback or forward path as a rotation matrix [7]. $\theta$ is the phase delay value obtained using the LMS algorithm that will be explained later in this article. This phase shift is implemented as shown in

$$
\begin{aligned}
\text { output }_{C H I}= & \cos (\theta) \cdot \text { Input }_{C H I} \\
& +\sin (\theta) \cdot \text { Input }_{C H Q} \\
\text { output }_{C H Q}= & -\sin (\theta) \cdot \text { Input }_{C H I} \\
& +\cos (\theta) \cdot \text { Input }_{C H Q}
\end{aligned}
$$

The output of Block I is applied to a DAC. The analog signal obtained at the output of the DAC is phase delayed by the circuits of the transmitter before reaching the output. The feedback signal obtained at the output of the transmitter is applied to an ADC and the vectorial product of the forward and feedback signals is obtained by Block II. Since the transmitter input signal is usually nondeterministic, a noise component is added to the signal. Because of the analog elements that compound the feedback path, the vectorial product of the forward and feedback signals is noisy. This noise introduces errors in the estimation of $\theta$. The aim of Block III, a low-pass filter, is to smooth and band-limit the noise generated by the vectorial product of the forward and feedback signals. This filter obtains an average value of the signal applied at its input (the vectorial product of forward and feedback signals), which helps achieve an accurate estimation of $\theta$. This filter is implemented using a second-order Butterworth approximation.

Subsequent DSP steps of the algorithm calculate the $\theta$ value that compensates the misalignment between forward and feedback signals. The implemented LMS algorithm in Block V

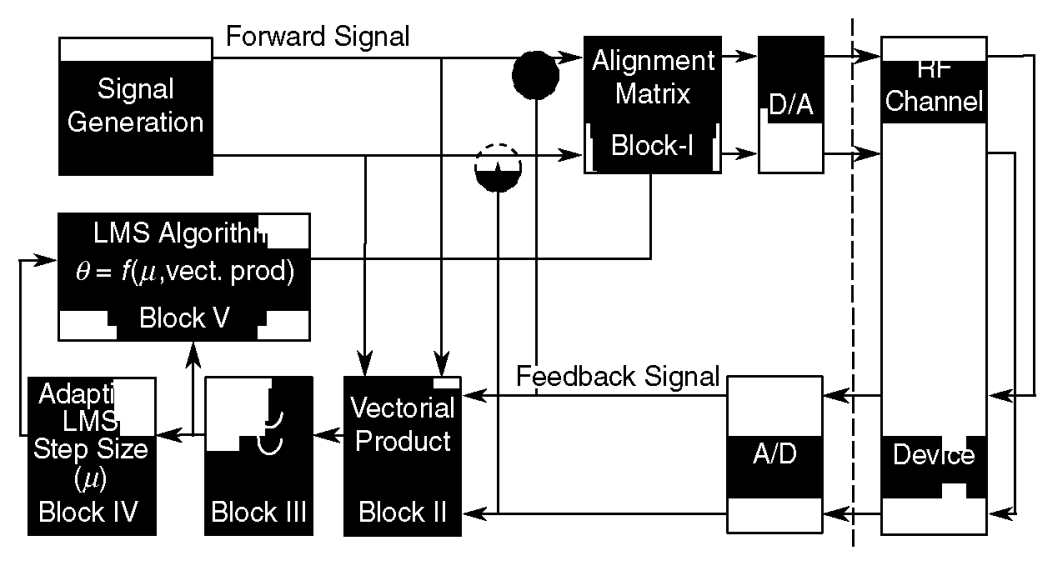

[FIG2] Block diagram of the phase alignment algorithm. 


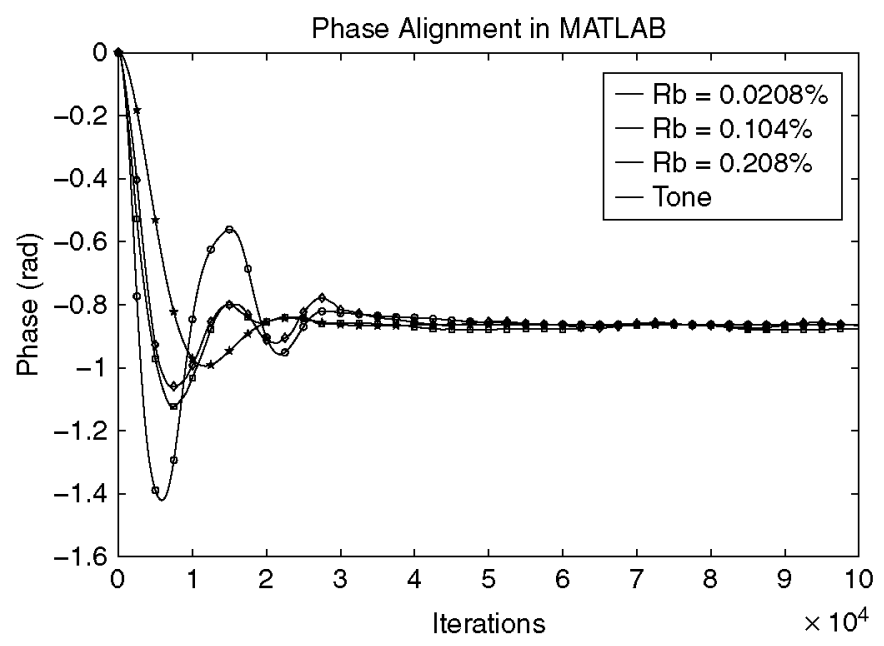

[FIG3] Phase alignment process in MATLAB.

searches for the gradient that zeros the vectorial product of forward and feedback signals, in such a way that the smaller the vectorial product, the closer $\theta$ is to its optimal value. Every $\theta$ value delivered by Block $V$ updates the matrix coefficients in Block I. This block uses (4) to calculate the phase difference between forward and feedback signals

$$
\theta[n+I]=\theta[n]+\mu \cdot V P,
$$

where $\theta[n+1]$ is the delay (radians) that will modify the matrix of the Block I and $\mu$ is the step-size of the LMS algorithm.

Block IV determines the convergence speed and the resolution of $\theta$ : Both the algorithm convergence speed and resid- ual error are determined by the step-size factor $\mu(0<\mu<1)$ of the LMS algorithm. Initially, when the algorithm starts, a high value is assigned to $\mu$; this way the LMS algorithm reaches a value close to the optimum one very quickly at the expense of accuracy. During the subsequent algorithm iterations, the value of $\mu$ is calculated on the basis of the result of the forward and feedback signals' vectorial product in such a way that the smaller the vectorial product, the smaller the value of $\mu$. The accuracy is improved after a period of time, since $\mu$ is smaller with each iteration until the vectorial product is zero. Equation (5) shows this algorithm

$$
\mu[n+1]=\mu[n]-V P \cdot 0.01,
$$

where 0.01 is a scale factor that determines the maximum reduction of $\mu$ every iteration and $V P$ is the vectorial product.

\section{ALGORITHM TESTING AND RESULTS}

To test the phase alignment algorithm, simulations and tests were performed on an EER RF power amplifier using a DSP linearization Cartesian feedback loop. First, the algorithm was simulated and tested using MATLAB. A single tone and a 16-quadrature amplitude modulation (QAM) signal (setting its carrier frequency at $0.4166 \%$ of the digital system sampling frequency) were used to perform testing. The algorithm was also tested for binary rates (Rb) $0.0208 \%, 0.104 \%$ and $0.208 \%$ of the sampling frequency. The phase difference between the forward and feedback signals of the Cartesian loop was set to 0.83775 rad. Results are depicted in Figure 3, which shows the phase misalignment estimation obtained by the algorithm from transient to steady state. The phase misalignment estimation $\theta$ accurately converges to steady state within less than 50,000 samples for all the signals used to test the system.

A DSP board based on the Analog Devices fixed point processor ADSP BF533 was used to test the algorithm in an EER linearized amplifier system. The results are shown in Figure 4. The time evolution of the estimated phase misalignment is similar to the simulated predictions obtained with MATLAB. The phase lag added by this hardware including DSP subsystems and RF circuits is time-variant and not completely known; however it was estimated about 0.83775 rad. The test results show that the performance of the algorithm is not different than the performance shown by MATLAB simulations concerning the time required to reach steady state. It must be noted that in this case the algorithm needed more samples to converge than the MATLAB simulations as a consequence of the hardware fixed-point arithmetic involved in the prototype implementation.

It is also observed that wider bandwidth signals experience higher error of the phase misalignment estimation and longer convergence times. This behavior 
results from the time delay between the forward and feedback signals in this algorithm and is translated to a constant phase misalignment at all frequencies.

\section{SUMMARY}

An adaptive algorithm to correct phase misalignments in Cartesian feedback linearization loops for power amplifiers has been presented. It yields an error smaller than $0.035 \mathrm{rad}$ between forward and feedback loop signals once convergence is reached. Because this algorithm enables a feedback system to process forward and feedback samples belonging to almost the same algorithm iteration, it is suitable to improve the performance not only of power amplifiers but also any other digital feedback system for communications systems and circuits such as all digital phase locked loops.

Synchronizing forward and feedback paths of Cartesian feedback loops takes a small period of time after the system starts up. The phase alignment algorithm needs to converge before the feedback Cartesian loop can start its ideal behavior. However, once the steady state is reached, both paths can be considered synchronized, and the Cartesian feedback loop will only depend on the loop parameters (open-loop gain, loop bandwidth, etc.). It means that the linearization process will also depend only on these parameters since the misalignment effect disappears.

Therefore, this algorithm relieves the power amplifier linearizer circuit design of any task required for solving phase misalignment effects inherent to Cartesian feedback systems. Furthermore, when a feedback Cartesian loop has to be designed, the designer can consider that forward and feedback paths are synchronized, since the phase alignment algorithm will do this task. This will reduce the simulation complexity. Then, all efforts are applied to determining the suitable loop parameters that will make the linearization process more efficient.

\section{ACKNOWLEDGMENTS}

This work was supported by the Spanish MEC funding (TEC2006-08210), Universidad Politecnica de Madrid, and Comunidad Autonoma de Madrid.

\section{AUTHORS}

Alejandro Gimeno-Martín (agimenomartin@gmail.com) is a member of the GIRA (Radio Engineering Group) research group at the Universidad Politecnica de Madrid, Spain. His research interests include digital processing techniques, power amplifiers, and radio frequency systems.
José M. Pardo-Martín (jmpardo@diac. upm.es) is a professor at the Polytechnic University of Madrid and a researcher in the GIRA research group. His interests include digital processing techniques, radio-software, and embedded systems.

Francisco J. Ortega-González (fjortega@) diac.upm.es) is a professor at the Polytechnic University of Madrid and leads the GIRA research group. His interests include power electronic and high frequency systems.

\section{REFERENCES}

[1] M. Joohansson and L. Sundström, "Linearization of RF multicarrier amplifiers using Cartesian feedback," IEE Electron. Left., vol. 30, no. 14, pp.
1110-1112, July 1994.

[2] C. Tassin, P. Garcia, J. B. Bégueret, R. Toupé, Y. Deval, and D. Belot, "A mixed-signal Cartesian feedback linearization system for a zero-IF WCDMA transmitter handset IC," in Proc. Ph.D. Research in Microelectronics and Electronics, July 2005, vol. 2, pp. 59-62.

[3] J. L. Dawson and T. H. Lee, "Automatic phase alignment for a fully integrated Cartesian feedback power amplifier system," IEEE J. Solid State Circuits, vol. 38, pp. 2269-2279, Dec. 2003.

[4] M. Johansson and T. Mattsson, "Transmitter linearization using Cartesian feedback for linear TDMA modulation," in Proc. 41st IEEE Vehicular Technology Conf., May 1991, pp. 439-444.

[5] J. L. Dawson and T. H. Lee, "Cartesian feedback for RF power amplifier linearization," in Proc. American Control Conf., July 2004, vol. 1, pp. 361-366.

[6] J. L. Dawson and T. H. Lee, "Automatic phase alignment for high bandwidth Cartesian feedback power amplifier," in Proc. Radio and Wireless Conf., 2000, pp. 71-74.

[7] S. I. Mann, M. A. Beach, and K. A. Morris, "Digital baseband Cartesian loop transmitter," Electron. Lett., vol. 37, pp. 1360-1361, Oct. 2001. 